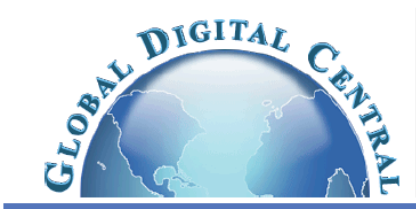

Frontiers in Heat and Mass Transfer

Available at www.ThermalFluidsCentral.org

\title{
CHARACTERIZATION OF THE RHEOLOGY AND CURE KINETICS OF EPOXY RESIN WITH CARBON NANOTUBES
}

\author{
R. J. Johnson* and R. Pitchumani ${ }^{\dagger}$ \\ Advanced Materials and Technologies Laboratory, Department of Mechanical Engineering, Virginia Tech, \\ Blacksburg, Virginia 24061-0238, USA
}

\begin{abstract}
Much research is currently being performed with carbon nanotube additives to neat resin systems to enhance properties such as thermal and electrical conductivity, strength, modulus and damping. Fabrication of parts based on carbon nanotube filled resin systems requires information on their cure kinetics and rheology, which has been relatively less studied so far. This work presents an extensive experimental study that systematically characterizes the cure kinetics and viscosity as a function of degree of cure and temperature of EPON 815C/EPICURE 3274 epoxy resin system laden with carbon nanotubes. Studies are conducted to determine the effects of the carbon nanotube loading and morphology on the cure reaction as well as the viscosity of the resin system in the early stages of the cure process, as is relevant in most processing applications. The experimental measurements are presented as generalized correlations as functions of the carbon nanotube parameters, carbon nanotube loading, temperature, degree of cure, and shear rate.
\end{abstract}

Keywords: chemorheology, nanocomposites, nanofluids, viscosity

\section{INTRODUCTION}

Nanocomposites based on a thermosetting resin reinforced with nanofillers such as nanoclays or carbon nanotubes have been studied quite extensively in recent years, for their promise of significantly improved properties. Specifically, nanocomposites with carbon nanotubes have been reported to reduce thermal expansion coefficient (Xu et al., 2006) while increasing thermal and electrical conductivities (Xu et al., 2006; Breuer and Sundararaj, 2004), modulus (Breuer and Sundararaj, 2004; Schadler et al., 1998), durability (Ajayan et al., 2000; Breuer and Sundararaj, 2004) and strength (Ajayan et al., 2000; Schadler et al., 1998; Breuer and Sundararaj, 2004), while nanocomposites filled with nanoclays have been shown to improve toughness ( $\mathrm{Li}$ et al., 2005), increase the heat distortion temperature (Kojima et al., 1993b,a) and reduce the apparent activation energy of the cure reaction (Ton-That et al., 2004). Motivated by these property enhancements, use of nanocomposites has been proposed for target applications such as electrostatically dissipative materials (Smith Jr. et al., 2004), light weight aerospace structural composites (Njuguna and Peilichowski, 2004), and vibration damped structural composites (Zhou et al., 2004; Koratkar et al., 2005; Johnson, 2007).

Many of the studies reported in the literature have focused on quantifying the properties of the nanocomposites as a function of the nanofiller morphology and loading. Ajayan, et al., (Ajayan et al., 2000; Schadler et al., 1998) studied the use of single-walled and multi-walled carbon nanotubes as reinforcement in an epoxy matrix using Raman spectroscopy to measure the strain held by a carbon nanotube under load. $\mathrm{Xu}$, et al., (Xu et al., 2006) measured the effects of single-walled carbon nanotubes on the thermal properties of a thermoplastic composite and found reductions in the coefficient of thermal expansion and increased thermal conductivity due to carbon nanotubes. Kim et al., (Kim et al., 2005) studied the effects of oxidation of multi-walled carbon nanotubes on the electrical conductivity of a carbon nanotube-epoxy composite and found increased conductivity of the nanocomposite as long as no damage to the multiwalled carbon nanotubes occurred during oxidation.

Experimental studies have shown that the strength of carbon nanotube composites is lower than that predicted by theory (Breuer and Sundararaj, 2004), which may be due to the interfacial bond between the nanotube and the matrix. While one set of studies focus on improving the interface (Wang et al., 2006; Velasco-Santos et al., 2003), the weak interface has also been exploited for improving the damping characteristics of nanocomposites (Zhou et al., 2004; Koratkar et al., 2002; Rajoria and Jalili, 2005; Johnson, 2007). A large volume of literature also exists on studying the properties of nanocomposites reinforced with nanoclays (Njuguna and Peilichowski, 2004). Work on producing carbon nanotubes cost effectively has also been reported (Ohshima et al., 2001; Hofmann et al., 2004; Wang et al., 2002; Biró et al., 2003), with the objective of commercial fabrication which can lead to future widespread use of nanocomposites.

Although several studies have focused on the properties of nanocomposites, fabrication of nanocomposites has been less systematically investigated. It is envisioned that as the property advantages of nanocompos-

\footnotetext{
${ }^{*}$ Presently with ASML, Wilton, Connecticut, USA

${ }^{\dagger}$ Corresponding author. Email: pitchu@vt.edu; Phone: +1 5402311776
} 
ites become well-established, processing of these composites will receive greater attention in an attempt to fabricate nanocomposite parts in a commercially viable way. Fundamentally, design of the fabrication processes will require information on the viscosity and cure kinetics of thermosetting resin systems with nanofillers. The temperature- and cure-dependent viscosity as well as the cure kinetics of catalyzed thermosetting resin systems without reinforcements is reported for a variety of resin systems (Lee et al., 1982; Ramakrishnan et al., 2000; Han et al., 1986). However, the addition of nanofillers to the resin systems is expected to fundamentally alter their viscosity and cure kinetics: (1) the viscosity will not only be a function of temperature and the degree of cure, as in neat resin systems, but additionally of the shear rate, the nanofiller loading, and the nanofiller morphology; (2) the cure kinetics could be altered due to the presence of the nanofillers that are comparable in size to that of the crosslinking macromolecules, although whether the effect will be to promote or hinder the crosslinking rate needs to be established. Despite its fundamental significance, scant studies can be found in the literature on the characterization of the viscosity and cure kinetics of nanofilled resin systems.

Xie et al. $(2004,2005,2006)$ studied the cure kinetics characterization of epoxy resins reinforced with multiwalled carbon nanotubes and carbon nanofibes. They reported that the addition of multiwalled carbon nanotubes to epoxy resins led to an acceleration of the initial cure reaction rate whereas the time to the maximum reaction rate decreased with increasing carbon nanotube content (Xie et al., 2004). Furthermore, the activation energy for the cure reaction was found to decrease with increasing carbon nanotube content. On the other hand, the inclusion of carbon nanfibers in epoxy was reported to have only a negligible effect on the initial reaction rates and the time to maximum reaction rate (Xie et al., 2005, 2006). Abdalla et al. (2008) investigated the effect of carboxyl and fluorine surface modification of multiwalled carbon nanotubes on the cure behavior of epoxy resins with the modified carbon nanotubes, and reported that the activation energy and rate constant were not affected by the fluorine modification whereas the resins with carboxylated multiwalled carbon nanotubes exhibited an increase in the activation energy and a lower rate constant for the cure reaction. Fan and Advani (2007) measured the storage modulus, complex viscosity and the steady state viscosity of epoxy suspensions with multiwalled carbon nanotubes for different dispersion states, aspect ratios, concentrations and network structures, and orientation states. Zhamu et al. (2007) investigated the flow behavior and cure process of reactive graphitic nanofiber/epoxy materials, and reported viscosity models as function of temperature and time. Terenzi et al. (2008) reported on cure kinetics and rheological measurements for a 1 wt.\% double walled carbon nanotube/epoxy system, and concluded that the addition of the carbon nanotubes led to speeding up of the cure reaction.

The objective of the present study is to systematically characterize the viscosity and cure kinetics of a carbon nanotube filled catalyzed epoxy resin system, toward establishing the fundamental information needed for processing of nanocomposites based on these materials. To this end, experimental measurements are reported on the effects of carbon nanotube morphology (single-walled and multi-walled), aspect ratio, and weight percentage in the resin on the viscosity and cure kinetics. Based on the experimental data, empirical correlations are developed for the first time, relating the viscosity as a function of the shear rate, the carbon nanotube parameters, the degree of cure and the temperature, and the cure rate as a function of the degree of cure, the temperature, and the carbon nanotube parameters. The empirical correlations will serve as basis for further studies on processing of nanocomposites. The experimental characterization studies are detailed in Section 2, followed by presentation of the results of the study and the correlations in Section 3.
Table 1 Ranges of carbon nanotube parameters considered in the study.

\begin{tabular}{|c|c|c|c|c|c|}
\hline No. & $\begin{array}{l}\text { CN } \\
\text { wt. \% }\end{array}$ & CN Type & $\begin{array}{l}\text { Diameter } \\
{[\mathrm{nm}]}\end{array}$ & $\begin{array}{l}\text { Length } \\
{[\mu \mathrm{m}]}\end{array}$ & $\begin{array}{c}\text { Aspect Ratio } \\
\text { Range }\end{array}$ \\
\hline 1 & 0.0 & - & - & - & - \\
\hline 2 & 0.1 & & & & \\
\hline 3 & 0.3 & MWCN & $30 \pm 15$ & $1-5$ & $22-333$ \\
\hline 4 & 0.5 & & & & \\
\hline 5 & 0.1 & & & & \\
\hline 6 & 0.3 & MWCN & $15 \pm 5$ & $1-5$ & $50-500$ \\
\hline 7 & 0.5 & & & & \\
\hline 8 & 0.1 & & & & \\
\hline 9 & 0.3 & MWCN & $30 \pm 15$ & $5-20$ & $111-1,333$ \\
\hline 10 & 0.5 & & & & \\
\hline 11 & 0.1 & & & & \\
\hline 12 & 0.3 & MWCN & $15 \pm 5$ & $5-20$ & $250-2,000$ \\
\hline 13 & 0.5 & & & & \\
\hline 14 & 0.1 & & & & \\
\hline 15 & 0.3 & SWCN & $<2$ & no spec. & - \\
\hline 16 & 0.5 & & & & \\
\hline 17 & 0.1 & & & & \\
\hline 18 & 0.3 & pSWCN & $<2$ & $0.5-120$ & $250-60,000$ \\
\hline 19 & 0.5 & & & & \\
\hline
\end{tabular}

\section{EXPERIMENTAL STUDIES}

A systematic experimental study was performed to determine the cure kinetics and viscosity of EPON 815C resin catalyzed with EPICURE 3274 curing agent and containing varying amounts, morphologies, and aspect ratios of carbon nanotube fillers. Carbon nanotubes of three types were investigated: multi-walled, research grade ( $>95 \%$ purity), carbon nanotubes (MWCN) supplied by NanoLab (Newton, MA), and singlewalled carbon nanotubes (SWCN) and purified single-walled carbon nanotubes (pSWCN), both supplied by SES Research (Houston, TX). For each type, different combinations of diameters, lengths, and weight percentages were considered. Table 1 enumerates the various combinations resulting in 18 carbon-nanotube-filled resin-catalyst samples and one sample with no carbon nanotubes, for a total of 19 samples. As seen in Table 1 , the MWCN diameters were nominally $15 \pm 5 \mathrm{~nm}$ or $30 \pm 15 \mathrm{~nm}$ and their nominal lengths were either $1-5 \mu \mathrm{m}$ or $5-20 \mu \mathrm{m}$. The SWCN used in the study were produced using the arc process and had a purity of 20$40 \%$ (other materials included metal catalyst and amorphous carbon and carbon nanoparticles); their nominal diameter was ranged from 1.2-1.4 $\mathrm{nm}$ and their nominal length was in the range of $2-5 \mu \mathrm{m}$. The pSWCN fillers were treated to open their ends, and had a purity of up to $75 \%$. Table 1 also lists the range of aspect ratios based on the nominal length and diameter values, for reference in the analysis of the results of the experiments. For each combination of carbon nanotube morphology, length, and diameter, three weight percentages of $0.1 \%, 0.3 \%$, and $0.5 \%$ were considered. The sample preparation and experimental characterization are described in the following subsections.

\subsection{Sample Preparation}

Samples for both cure kinetics and viscosity experiments were prepared by a process of weighing, hand mixing, ultrasonic processing, and degassing. First, appropriate amounts of carbon nanotubes and EPON 815C resin were weighed using an Ohaus Explorer Pro precision balance (Ohaus Corporation, Pine Brook, NJ) and hand mixed in a sample vial. Next, the carbon nanotubes were dispersed in the resin using a Cole-Parmer 500 watt ultrasonic processor with a $1 / 4$ in. tapered microtip. The ultrasonic technique was chosen based on its effectiveness in dispersing carbon nanotubes in resin systems, as reported in previous studies (Fan and Advani, 2007). The microtip of the ultrasonic processor, driven by a 

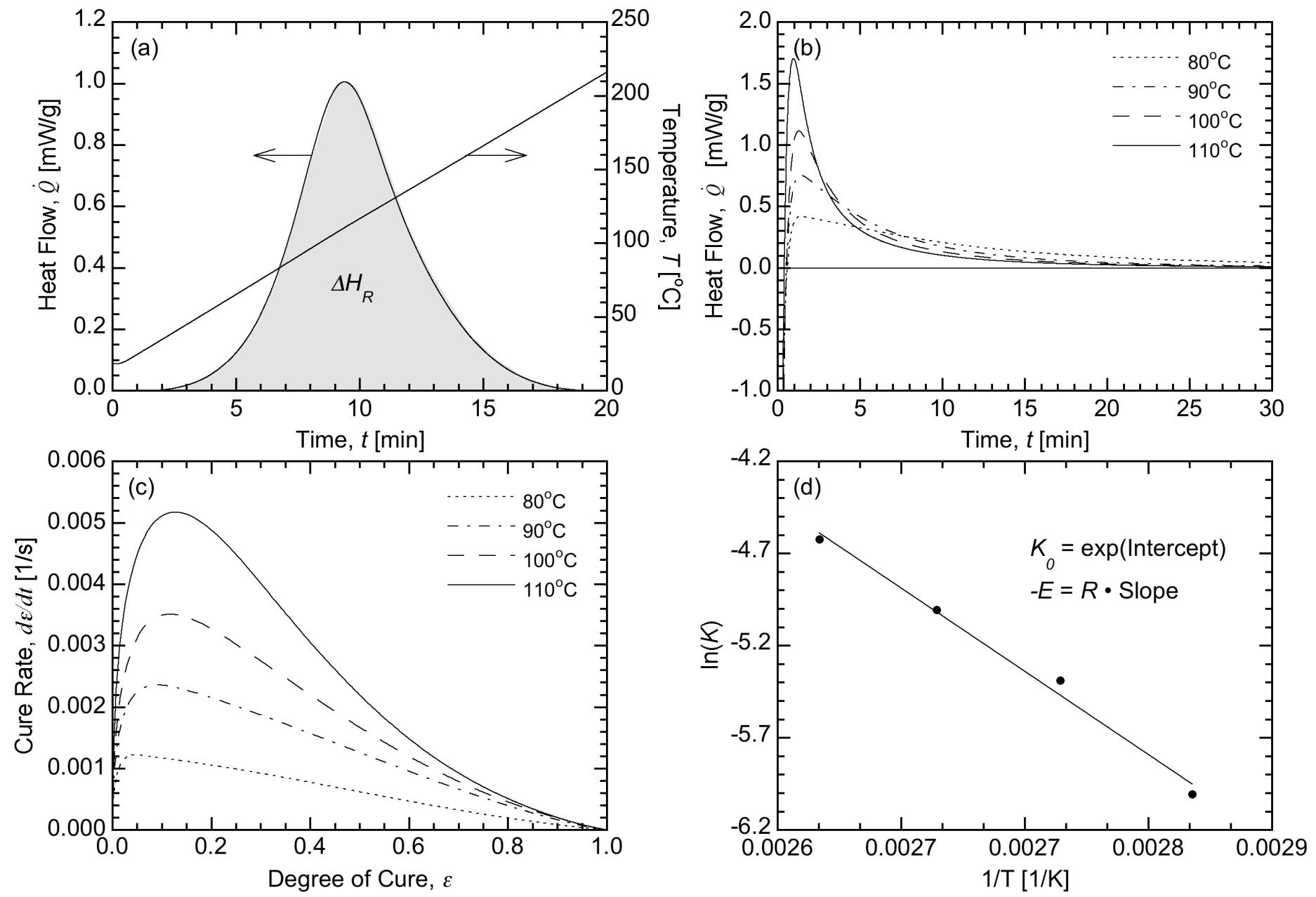

Fig. 1 (a) Exothermic heat flow with time during a nonisothermal dynamic scan in a differential scanning calorimetry experiment on Sample 6 (Table

1); (b) heat flow during isothermal scans at four different temperatures measured using differential scanning calorimetry on Sample 6 (Table 1);

(c) conversion of the measured data in (b) to cure rate variation with the degree of cure; and (d) determination of the corresponding parameters of the cure kinetics equation (Eq. 1).

piezoelectric device, oscillated at $20 \mathrm{kHz}$ and generated pressure waves that deagglomerated carbon nanotube clusters held together by van der Walls forces thereby promoting uniform dispersion. The sample vial was partially submerged in water bath to maintain a low temperature during processing and to avoid heat build-up that could degrade the resin. The end of the tapered microtip was submerged in the sample, taking care to ensure that the tip did not contact the sample vial and each sample was sonified for 2 hours at the minimum amplitude setting of $20 \%$ in a pulsed mode with the input being alternated on and off for one second duration each. Sonification in the pulsed mode further reduced the energy input rate to the sample to allow time for heat to dissipate to the water bath, and ensured that the sample temperature never exceeded $50^{\circ} \mathrm{C}$ during processing. Finally, the sample was degassed in a vacuum chamber at $25 \mathrm{in}$. $\mathrm{Hg}$ for 30 minutes. Immediately prior to each individual experiment resin (with dispersed carbon nanotubes) and curing agent were measured using syringes with $10 \mu \mathrm{L}$ divisions and thoroughly hand mixed at a 2.19:1 ratio (by volume) to make approximately $2 \mathrm{~mL}$ of total sample. These prepared samples were utilized in the cure kinetics and viscosity experiments as outlined in the following sections.

\subsection{Cure Kinetics Characterization}

The cure kinetics of the catalyzed epoxy resin with and without carbon nanotubes were characterized using a differential scanning calorimeter
(DSC) (Model: DSC 2920 from TA Instruments, New Castle, DE). For each experiment, a small amount of the epoxy-curing agent-carbon nanotube mixture was placed in a sample pan, crimp-sealed, and weighed. The samples were tested under a dynamic (nonisothermal) condition as well as several isothermal conditions. In the dynamic measurements, the sample was placed in the DSC chamber, and the temperature was ramped at a rate of $10^{\circ} \mathrm{C} / \mathrm{min}$ from room temperature to $225^{\circ} \mathrm{C}$, where the reaction was seen to be complete. For each sample subject to an isothermal scan, a secondary nonisothermal scan was conducted to ensure that there was no residual reaction, thus confirming that the sample was cured completely under the isothermal conditions. The exothermic heat flow rate during the experiment was measured as a function of time, as illustrated in Fig. 1(a) for an example case of Sample 6 in Table 1; the heat flow rate initially increases rapidly, reaches a peak and drops to zero. The area under the bell-shaped heat flow curve, denoted by the shaded region in Fig. 1(a), represents the heat of the reaction, $\Delta H_{R}$.

Isothermal measurements of heat flow were performed on the 19 different samples at four different target temperatures of $80^{\circ} \mathrm{C}, 90^{\circ} \mathrm{C}$, $100^{\circ} \mathrm{C}$, and $110^{\circ} \mathrm{C}$ to determine the temperature-dependent reaction rate. In these experiments, the DSC chamber was preheated to the desired isothermal temperature before placing the sample in the chamber for the heat flow measurements. Figure 1(b) presents the measured heat release for Sample 6 for the four different temperatures, where it is noted that 

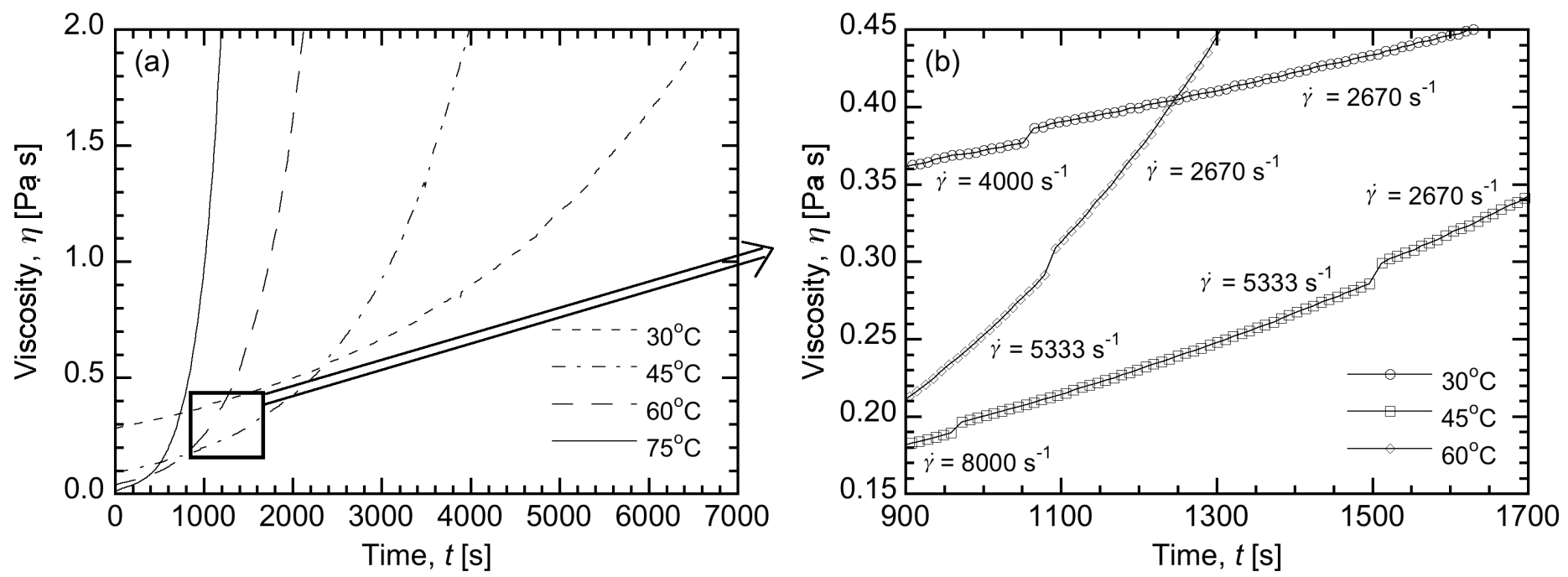

Fig. 2 (a) Variation of the measured viscosity with time at four different temperatures for Sample 6 (Table 1) and (b) a close-up view of the boxed region in (a) to illustrate the shear-rate-dependence of the viscosity.

the reaction gets progressively faster with higher peak heat flow values as the temperature increases. Note that the brief endotherm (negative heat flow values) at the beginning of the measurements corresponds to the initial energy flow into the sample when it is placed in the isothermal DSC chamber; this data is disregarded in the exothermic heat flow calculations and the time for the cure reaction was measured from the point when the heat flow crossed the value of zero. It was also verified that the area under each of the isothermal heat flow curves was close to the heat of the reaction value obtained from the dynamic scan data. The isothermal heat flow measurements were converted to cure rate $(d \varepsilon / d t)$ values by dividing the heat flow rate, $\dot{Q}$, [Fig. 1(b)] by the heat of the reaction, $\Delta H_{R}$. Integrating the time-dependent cure rate plot from the initial time $(t=0)$ to any time, $t$, yields the degree of cure, $\varepsilon(t)$. Figure $1(\mathrm{c})$ presents the variation of cure rate with the instantaneous degree of cure, $\varepsilon(t)$, based on the isothermal heat flow plots in Fig. 1(b).

The plots of cure rate variation with the degree of cure were expressed in terms of an empirical correlation of the form below, which has been used to describe the cure kinetics of EPON 815C/EPICURE 3274 system previously (Ramakrishnan et al., 2000)

$$
\frac{d \varepsilon}{d t}=\underbrace{K_{0} \exp \left(\frac{-E}{R T}\right)}_{K} \varepsilon^{m}(1-\varepsilon)^{n}
$$

For each temperature, the values of $K, m$, and $n$ were determined through a nonlinear regression of the corresponding data in Fig. 1(c), using the following weight function, $W$, that emphasized fitting of the rapidly changing data near the peak cure rate.

$$
W=\frac{1}{\max (\dot{\varepsilon})}(\max (\dot{\varepsilon})-\dot{\varepsilon})+\varepsilon
$$

As is commonly observed for autocatalytic reactions (Opaliki et al., 1996; Ramakrishnan et al., 2000; Han et al., 1986; Kamal and Sourour, 1973), the values of $m$ and $n$ were determined to be such that $m+n$ $=2$. From the values of $K$ for the four different temperatures, the frequency factor, $K_{0}$, and the activation energy for the cure reaction, $E$, were obtained from a semi-log plot of $K$ with respect to the inverse of the absolute temperature (in Kelvin), as shown in Fig. 1(d); the slope of a linear regression fit through the data in Fig. 1(d) represents $-E / R$ and the intercept on the vertical axis denotes $\ln \left(K_{0}\right)$.
The kinetics parameters, $K_{0}, E, m$ and $n$ were determined for all the samples listed in Table 1 following the methodology presented above. The effect of the carbon nanotube parameters on the cure kinetics is discussed in the section on presentation of results.

\subsection{Viscosity Characterization}

Viscosity of the 19 samples in Table 1 was measured dynamically throughout the cure process using a Brookfield CAP 2000+ cone-and-plate viscometer (Brookfield Engineering, Middleboro, MA) with the goal of characterizing the viscosity as a function of degree of cure, temperature, shear rate, carbon nanotube filler content, morphology, and carbon nanotube aspect ratio. The viscometer has a temperature controlled plate to allow for the required range of elevated temperature, isothermal tests. The CAP01 cone with a $0.45^{\circ}$ cone angle and a $1.511 \mathrm{~cm}$ radius was used for the measurements which allowed for a small sample size $(<100 \mu \mathrm{L})$, thereby reducing the amount of carbon nanotubes used in the experiments. The cone angle of $0.45^{\circ}$ corresponds to a central portion of the circular plate area being subject to possible dry contact between the cone and plate through a carbon nanotube oriented on its end. This central area of possible dry contact was calculated to be $2.8 \%$ of the total plate area for the largest carbon nanotube and less than $0.007 \%$ for the smallest carbon nanotube length considered in the experiments. The minimal values of the dry contact area suggest that the viscosity measurements were not significantly affected by the carbon nanotube interactions with the surfaces of the viscometer cone and plate.

The epoxy-carbon-nanotube sample prepared following the steps in Section 2.1 was hand-mixed with the curing agent, and a drop of the mixture was immediately placed on the preheated viscometer plate. Four isothermal temperatures of $30,45,60$ and $75^{\circ} \mathrm{C}$ were considered in the experiments. The shear rate for the experiment was set by adjusting the rotational speed of the cone. An initial rotational speed was set at the beginning of each measurement run based on the torque being within the range of the viscometer. As the resin mixture cures, its viscosity and, correspondingly, the torque increase. In order to keep the torque values within the limits of the viscometer, the rotational speed was reduced periodically during each measurement run. Thus, each run consisted of a series of piecewise constant shear rates, and each jump in the shear rate provided for extracting the shear-dependence of the viscosity. Data collection was automated and carried out using the Brookfield CapCalc software (Brookfield Engineering, Middleboro, MA) to record the history of viscosity, shear rate, and temperature during each measurement run. 
Figure 2 shows the results of these measurements for the example case of Sample 6 in Table 1 with 0.3 wt. $\% 15 \mathrm{~nm}$ diameter $1-5 \mu \mathrm{m}$ length multi-walled carbon nanotube filler. Figure 2(a) shows the expected trend of viscosity decreasing with increasing temperature at zero time (or zero cure) and the increase in viscosity as a result of increasing cure (increasing time). Figure 2(b) represents a magnified image of the boxed region in Fig. 2(a) and illustrates the piecewise constant shear rate values in the time range of the measurements shown. Note the jump in measured viscosity at times of discrete changes in the shear rate depicting the non-Newtonian shear-thinning characteristic of the carbon nanotube filled resin system. The shear-thinning rheology of resins with carbon nanotubes is consistent with the observations of Fan and Advani (2007) as well as the rheology of other particle-filled systems in the literature (Kordon et al., 1993). From the recorded temperature history, the corresponding degree of cure history was calculated using the cure kinetics model discussed in Section 2.2. Thus, the viscosity data were obtained as a function of time, temperature, shear rate, and degree of cure.

An empirical correlation for viscosity as a function of temperature and degree of cure suggested for epoxy resin systems in the literature (Advani, 1994) is modified here to include additionally the power law dependence on shear rate, and is expressed as follows:

$$
\eta=\underbrace{A \exp \left(\frac{E_{\eta}}{R T}+B \varepsilon\right)}_{M} \dot{\gamma}^{n_{1}-1}
$$

Here, $\eta$ is the viscosity of the resin system, $T$ is the temperature in Kelvin, $\varepsilon$ is the degree of cure discussed in Section 2.2, $\dot{\gamma}$ is the shear rate, $E_{\eta}$ is the activation energy, and $R$ is the universal gas constant. The term denoted as $M$ represents the consistency index as a function of temperature and degree of cure, in which $A$ and $B$ are empirical constants. The term $n_{1}$ represents the power law exponent, which is expected to be less than unity based on the shear-thinning characteristic observed in Fig. 2(b).

At each instance the shear rate was changed (see Fig. 2(b)) a value of the power law exponent, $n_{1}$, was computed assuming the change in viscosity across the jump in shear rate is purely attributable to the change in shear rate. Values of $n_{1}$ were seen to be similar for all instances of shear rate change corresponding to a particular sample and the average of these values was used as $n_{1}$ for the correlation. The consistency index, $M$, was then determined for each data point corrispondng to a particular sample and temperature, based on a re-expression of Eq. 3 as:

$$
M=\frac{\eta}{\dot{\gamma}^{n_{1}-1}}
$$

This yielded consistancy index, $M$, values for a particular sample as a function of temperature, $T$, and degree of cure, $\varepsilon$. Figure 3(a) shows a semi-log plot of the consistency index as a function of the degree of cure for the four studied temperatures. Note that the least squared linear regression of the data (solid line) has a constant slope for all temperatures, which corresponds to $B$, and the intercepts correspond to $\ln (A)+\frac{E_{\eta}}{R T}$. The values of $A$ and $E_{\eta}$ are, in turn, determined by a linear least-squared regression fit of the intercepts as a function of the inverse of the temperature as shown in Fig. 3(b) where $A$ is the exponential of the intercept and $E_{\eta}$ is obtained as the product of the universal gas constant and the slope.

The values of $A, B, E_{\eta}$, and $n_{1}$ determined through the foregoing approach completely characterize the viscosity as a function of cure and temperature of the resin system with carbon nanotube fillers. The measurements were conducted for all the samples listed in Table 1, and the parameters were determined. The effects of the carbon nanotubes on the viscosity are discussed in the following section.

\section{RESULTS AND DISCUSSION}

The cure kinetics and viscosity of the resin systems filled with carbon nanotubes are characterized by their respective sets of parameters: $\Delta H_{R}$,
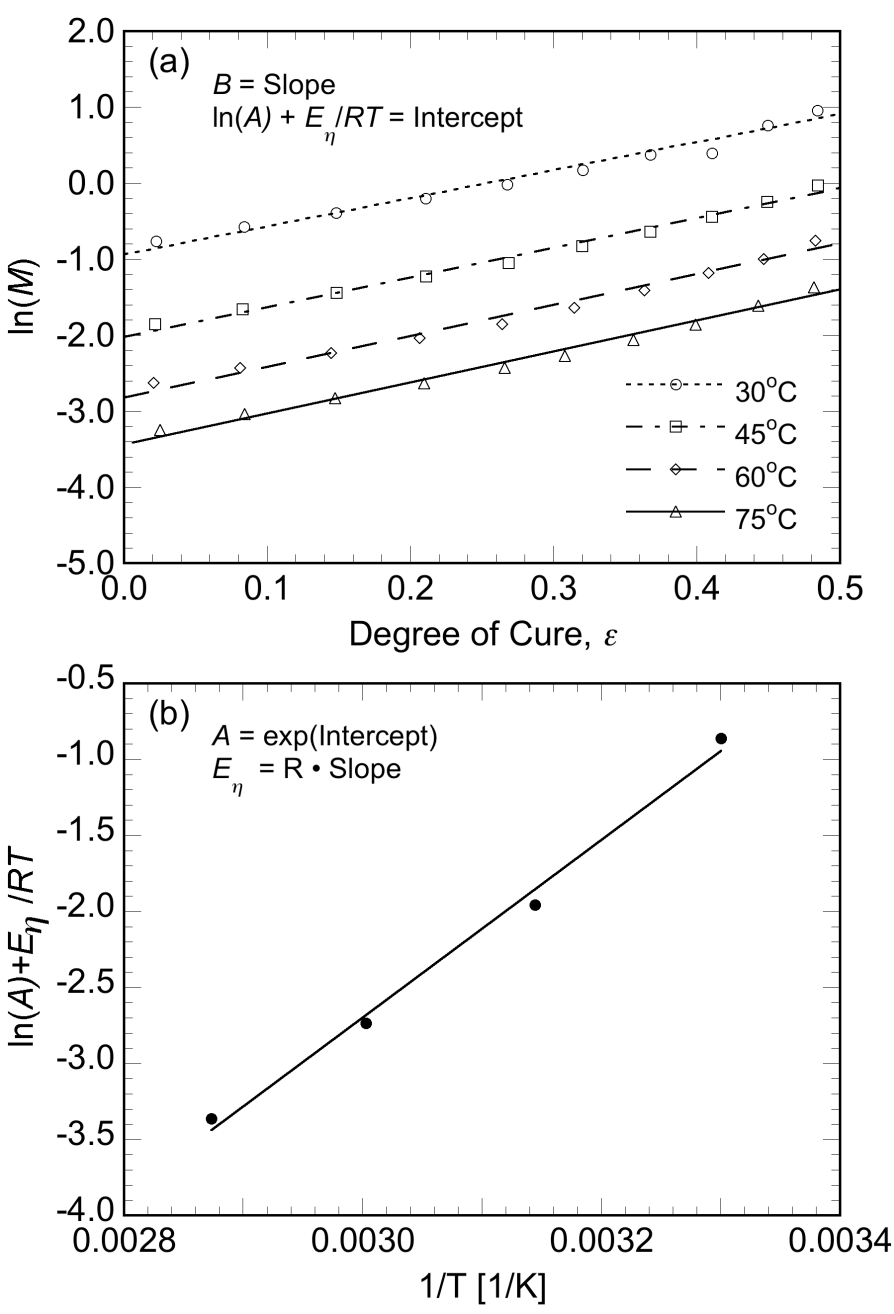

Fig. 3 Determination of the parameters, (a) $B$ and (b) $A$ and $E_{\eta}$, of the viscosity equation.

$K_{0}, E, m$ and $n$; and $A, B, E_{\eta}$, and $n_{1}$. The variation of these parameters with carbon nanotube loading, morphology, and aspect ratio is examined in this section. Furthermore, the correlations for cure kinetics (Eq. 1) and viscosity (Eq. 3) are generalized to incorporate the effects of the carbon nanotube parameters.

\subsection{Cure Kinetics}

The differential scanning calorimetric measurements on the 19 samples resulted in the heat of the reaction values, $\Delta H_{R}$, from about $315 \mathrm{~J} / \mathrm{g}$ to about $360 \mathrm{~J} / \mathrm{g}$, with no distinct trend with respect to the carbon nanotube loading, aspect ratio, or morphology. This suggests that the carbon nanotubes do not affect the exothermic heat release from the reaction, and an average value of $349.6 \mathrm{~J} / \mathrm{g}$ from the 19 experiments is taken to be the heat of the reaction for the samples. Similarly, the presence of the carbon nanotubes was found to have minimal effect on the shape parameters, $m$ and $n$, which were taken to be the values corresponding to the neat resin sample (Sample 1 in Table 1): $m=0.207$ and $n=1.793$.

Figure 4(a) presents the variation of the cure reaction rate constant, $K$, with the carbon nanotube loading for the four different temperatures studied, based on the measurements on the 19 samples. The various markers at each temperature and carbon nanotube loading correspond to the different aspect ratios and morphologies considered. Since there was no discernible trend with respect to the aspect ratio or morphology, an average of the rate constants for all the aspect ratios and morphologes 

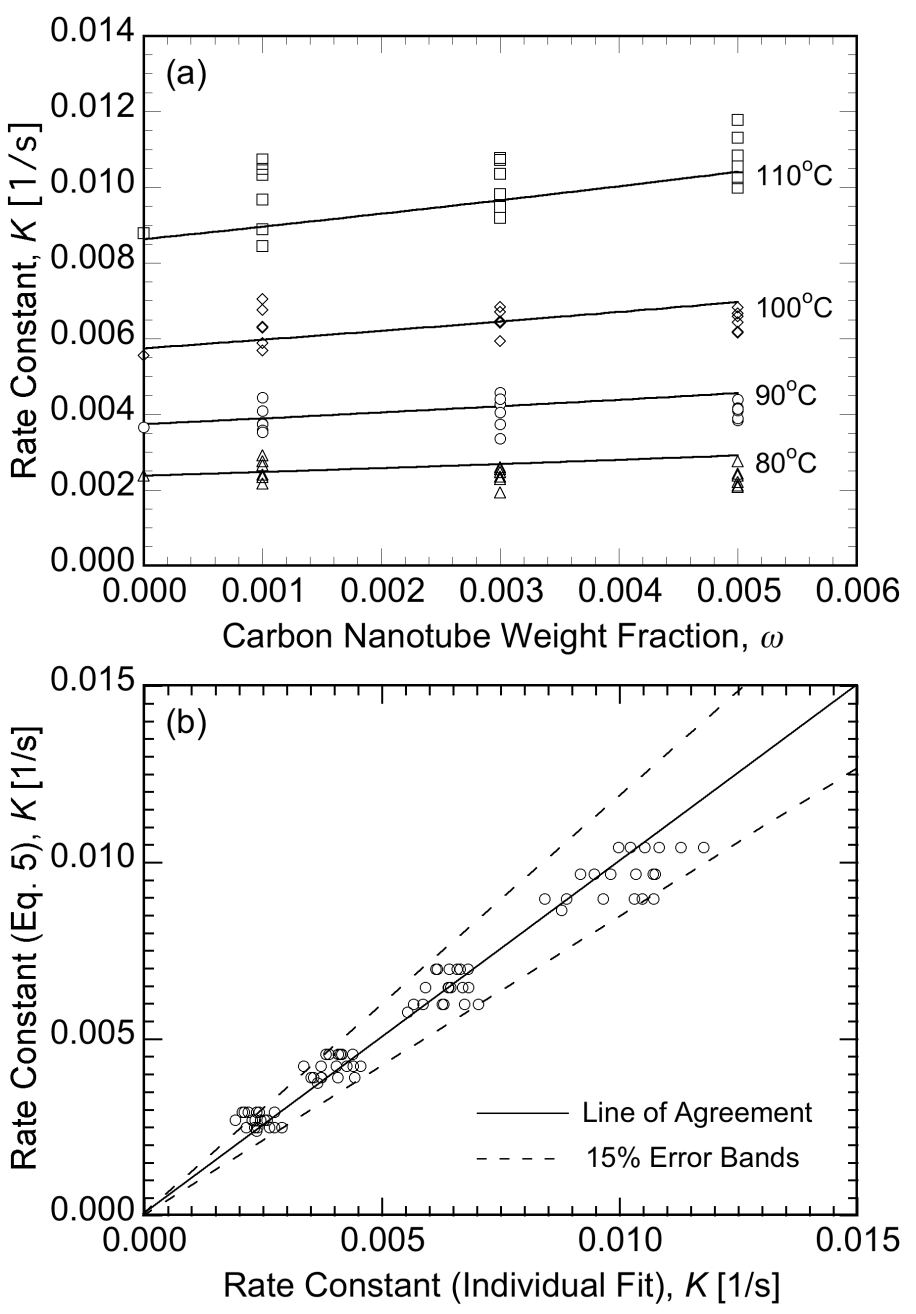

Fig. 4 (a) Variation of the rate constant, $K$, with the carbon nanotube weight fraction at four different temperatures, and (b) comparison of the rate constants predicted by the generalized cure kinetics equation (Eq. 5) with those measured on the individual samples, for all the 19 samples in Table 1.

corresponding to each combination of temperature and carbon nanotube loading was used to investigate the trend in the reaction rate with increasing carbon nanotube loading. It is seen that the reaction rate is generally enhanced by the addition of carbon nanotubes, and that the magnitude of the enhancement is more pronounced at the higher temperatures. The temperature-dependent increase in the reaction rate is modeled in this study through a decrease in the activation energy for the reaction with increasing carbon nanotube content while keeping the frequency factor $K_{0}$ to be independent of the carbon nanotube loading. Accordingly, a modification to the standard cure kinetics model (Eq. 1) is proposed to describe the effect of the carbon nanotube loading on the reaction kinetics as follows:

$$
\frac{d \varepsilon}{d t}=\underbrace{K_{0} \exp \left(\frac{-E(1-\omega)^{2.5}}{R T}\right)}_{K} \varepsilon^{m}(1-\varepsilon)^{n}
$$

where $K_{0}$ and $E$ are the frequency factor and the activation energy of a neat resin/catalyst sample without any added carbon nanotubes (Sample 1 in Table 1), which were determined to be $6.83 \times 10^{6} 1 / \mathrm{s}$ and -64.672 $\mathrm{kJ} / \mathrm{mol}$. In Eq. 5, $\omega$ represents the carbon nanotube loading expressed as a weight fraction. The values of $m$ and $n$ are those given earlier in this

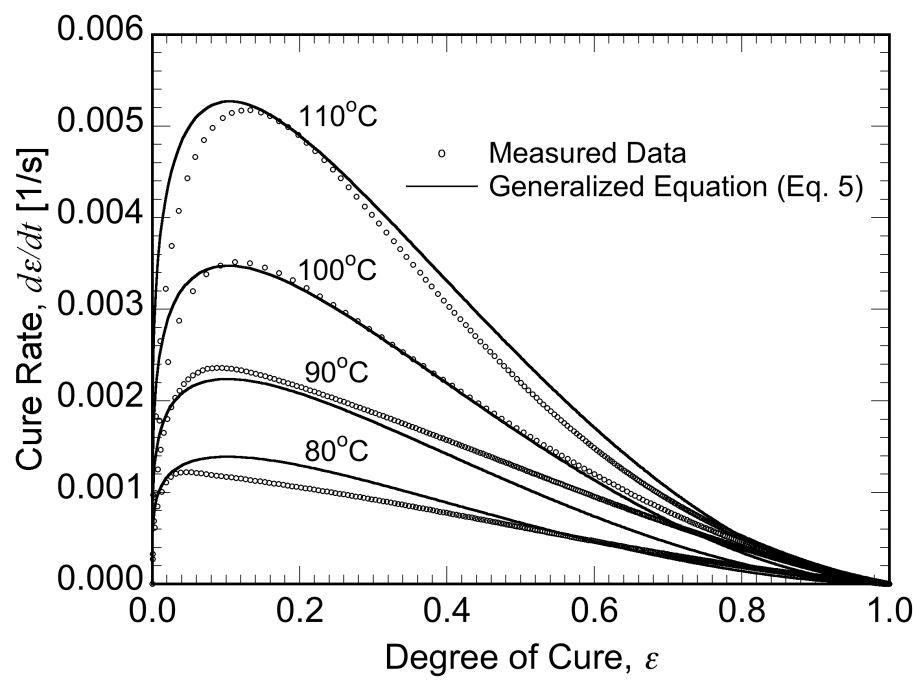

Fig. 5 Validation of the generalized cure kinetics model (Eq. 5) with the measured data for Sample 6 (Table 1) illustrating the high degree of model accuracy.

subsection. The term $E(1-\omega)^{2.5}$ in the modified cure kinetics relationship above is obtained through an empirical fit of the reaction rate data determined from experiments, and reflects the decrease in the activation energy with increasing carbon nanotube loading, a trend also reported for nanoclay filled systems (Ton-That et al., 2004). Furthermore it is seen that for $\omega=0$, the generalized equation defaults to the kinetics for an unfilled resin-catalyst system (Eq. 1).

Figure 4(b) compares the prediction of the rate constant using the expression for the term $K$ identified in Eq. 5 with the individually determined rate constants for all the samples studied. The solid line diagonal to the plot frame is the line of exact agreement and the dashed lines denote the $15 \%$ error bands. It is seen that the predictions of the rate constant obtained using the expression for $K$ in Eq. 5 are accurate to within $15 \%$ in almost all cases. The predictions of the cure rate, $\dot{\varepsilon}=d \varepsilon / d t$, obtained using the generalized correlation, Eq. 1, is shown in Fig. 5 for an example case with multi-walled carbon nanotube fillers (Sample 6 in Table 1). It is evident that the cure rate is also accurately predicted as a function of cure for all the temperatures. The correlation given by Eq. 5, and the associated parameter values, therefore, forms a reliable basis for modeling the cure of the carbon nanotube filled epoxy resin system considered in the study, incorporating the effects of the carbon nanotubes on the reaction.

\subsection{Viscosity}

The power law exponent, $n_{1}$, and the consistency index, $M$, including its constituent parameters, $A, B$, and $E_{\eta}$, in Eq. 3 were determined for all the 19 samples listed in Table 1 following the procedure outlined in Section 2.3. Based on the results for the various Samples with different carbon nanotube parameters, the power law exponent, $n_{1}$, was found to decrease with increasing concentration of the carbon nanotubes suggesting an increased shear-thinning behavior with increasing carbon nanotube content, as is commonly expected for particulate filled systems (Kordon et al., 1993). However, no distinct trend was observed with either the aspect ratio or the morphology of the nanotubes. The exponent was found to correlate well with the carbon nanotube weight fraction, $\omega$, as:

$$
n_{1}=1+30.3 \log _{10}(1-\omega)
$$

where the constant was determined through a least-squares fit of exponent values with $\omega$. Note that the form of the correlation is consistent with that used to represent the shear-thinning behavior with particulate volume fraction in ceramic slurries, reported in (Kordon et al., 1993), and 


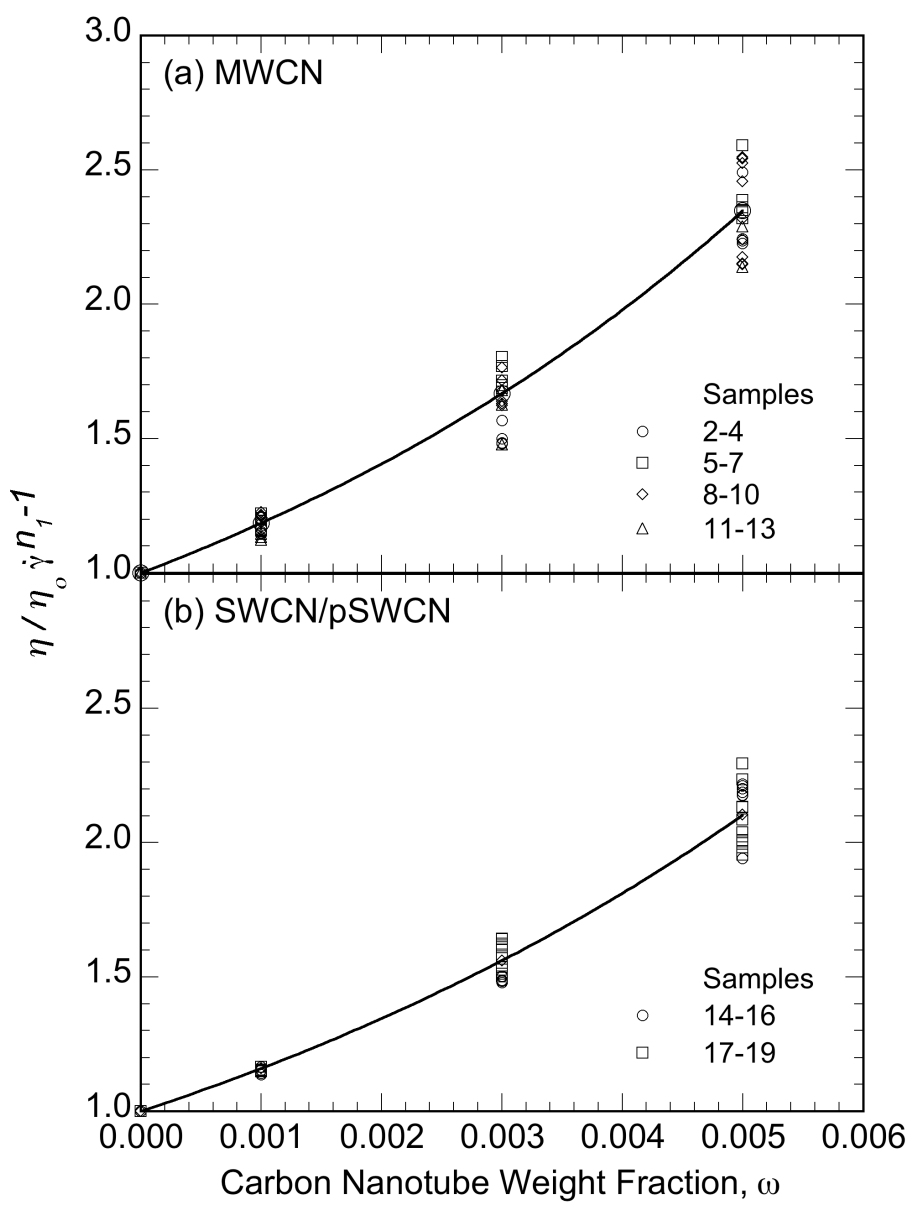

Fig. 6 Weight fraction dependence of the viscosity of (a) multi-walled carbon nanotubes (Samples 2-13 of Table 1) and (b) single-walled carbon nanotubes (Samples 14-19 of Table 1).

is such that the Newtonian behavior of unfilled catalyzed resin system is recovered for $\omega=0$.

The consistency index values of the 19 samples indicated an increase with the carbon nanotube weight fraction, $\omega$, for a given degree of cure, $\varepsilon$, and temperature, $T$. In order to characterize the weightfraction-dependence, the variation of $M$ with the weight fraction was represented as $M=\eta_{o} \cdot f(\omega)$, where $\eta_{o}$ is the viscosity of the unfilled resin-catalyst system (Sample 1 in Table 1), $\eta_{o}=A \exp \left(E_{\eta} / R T+B \varepsilon\right)$, and $f(\omega)$ is the dependence of the consistency index on the weight fraction of the carbon nanotubes, such that Eq. 3 can be expressed as: $M=$ $\eta_{o} \cdot f(\omega) \cdot \dot{\gamma}^{n_{1}-1}$. Figure 6(a) shows a plot of $f(\omega)$, the measured viscosity values for the multi-walled carbon nanotube samples (Samples 2-13 of Table 1) scaled with respect to $\eta_{o} \cdot \dot{\gamma}^{n_{1}-1}$, as a function of the weight fraction of the carbon nanotubes, $\omega$. In determining $f(\omega)$, the expression for $n_{1}$ given by Eq. 6 was used. Also included in Fig. 6(a) is Sample 1 corresponding to $\omega=0$, and the various markers for each weight fraction denote the different aspect ratios of the multi-walled carbon nanotubes.

The monotonically increasing trend of $f(\omega)$ was expressed using the function $\exp \left(K_{\eta} \omega /(1-\omega)\right)$, which mimics the form of the Mooney equation commonly used to model the rheology of particulate suspensions (Nielsen and Landel, 1994) with the modification that the carbon nanotube weight fraction, a more easily measurable quantity for carbon nanotubes, is used here instead of the particulate volume fraction in the Mooney equation. The solid line in Fig. 6 denotes this exponential form and is seen to correlate well with the measured data. The value of the coefficient, $K_{\eta}$, was determined through a least-squares regression of the data. A similar plot (Fig. 6(b)) for the set of single-walled carbon nanotube (SWCN and pSWCN) samples, Samples 14-19 in Table 1, was constructed to determine the corresponding value of the coefficient, $K_{\eta}$.

Based on the foregoing representation of the dependence of the consistency index and the power law exponent on the carbon nanotube parameters, the viscosity is expressed in a generalized form as:

$$
\eta=A \exp \left(\frac{E_{\eta}}{R T}+B \varepsilon+\frac{K_{\eta} \omega}{(1-\omega)}\right) \dot{\gamma}^{30.3 \log _{10}(1-\omega)}
$$

in which: $A=6.16 \times 10^{-11} \mathrm{~Pa} \cdot \mathrm{s} ; B=3.76 ; E_{\eta}=55.947 \mathrm{~kJ} / \mathrm{mol} ; K_{\eta}=$ 170 (MWCN) or 148 (SWCN and pSWCN). The coefficient, $K_{\eta}$, being different for the MWCN and the SWCN/pSWCN samples suggests the aspect ratio dependence of the viscosity. Since the aspect ratio values were not reliably known, no attempt was made to establish a quantitative relationship with the aspect ratio. Table 1 shows that the single-walled carbon nanotubes nominally had a higher aspect ratio range relative to the multi-walled carbon nanotubes. The lower $K_{\eta}$ value for SWCN/pSWCN points to a better alignment of the larger aspect ratio single-walled carbon nanotubes in the shear flow leading to a lower viscosity, although further studies are warranted for a conclusive determination.

The viscosity prediction by the generalized correlation in Eq. 7 is shown in Fig. 7(a) where the measured and predicted viscosity values are plotted as a function of time for Sample 6 in Table 1. The experimental data (dashed lines) correspond to the raw measurements from the viscometer. For each time corresponding to the experimental measurement, the degree of cure was computed using the generalized cure kinetics equation, Eq. 5, and was used along with the temperature and shear rate values in Eq. 7 to obtain the viscosity prediction. The viscosity values predicted by Eq. 7 are indicated by the solid lines in Fig. 7(a), for degree of cure, $\varepsilon<0.4$. Note that the predicted values match the experimental data well as a function of: temperature, as seen by the four isothermal curves, time (or equivalently degree of cure), as seen by the variation across the plot, and the shear rate, as seen by the discrete jumps in the viscosity values at the points of shear rate reduction in the experiment.

The overall accuracy of the general viscosity equation (Eq. 7) for all the samples and measurement conditions considered in this study is summarized in Fig. 7(b) where the predicted viscosity using Eq. 7 is plotted against the experimentally measured viscosity for degree of cure values less than 0.4. The solid diagonal line is the line of exact agreement and the dashed lines represent the $\pm 15 \%$ error bands. Figure 7 (b) represents over 25,000 measured viscosity data points, of which more than $90 \%$ fall within the $15 \%$ error bands. The generalized viscosity equation given Eq. 7 , therefore, represents a reliable viscosity model for evaluating the viscosity of the carbon nanotube filled epoxy resin system considered here. The generalized viscosity model given by Eq. 7 is demonstrated to be valid over the range of measurements considered in this study namely, carbon nanotube weight fraction up to $0.5 \%$; temperatures between $25-$ $75^{\circ} \mathrm{C}$; degree of cure below 0.4 (which represents pre-gelation conditions of the resin-catalyst system); and shear rate in the range 1,333-10,666 $\mathrm{s}^{-1}$. The primary objective of the present study was to investigate the chemorheology and the cure kinetics of resin systems with carbon nanotube suspensions, for use in composites processing applications, such as via liquid composite molding. To this end, carbon nanotube content of up to $0.5 \%$ was considered in the experimental studies and in the model development. The validity of the generalized cure kinetics model (Eq. 5) as well as the generalized viscosity model (Eq. 7) outside of the present range of the measurements may be established in a future study.

\section{CONCLUSIONS}

An extensive experimental study was conducted to determine the cure kinetics and viscosity characteristics of EPON 815C/EPICURE 3274 resincatalyst system with carbon nanotube fillers, over a range of carbon nanotube loading, morphology, and aspect ratios. The study revealed that the 

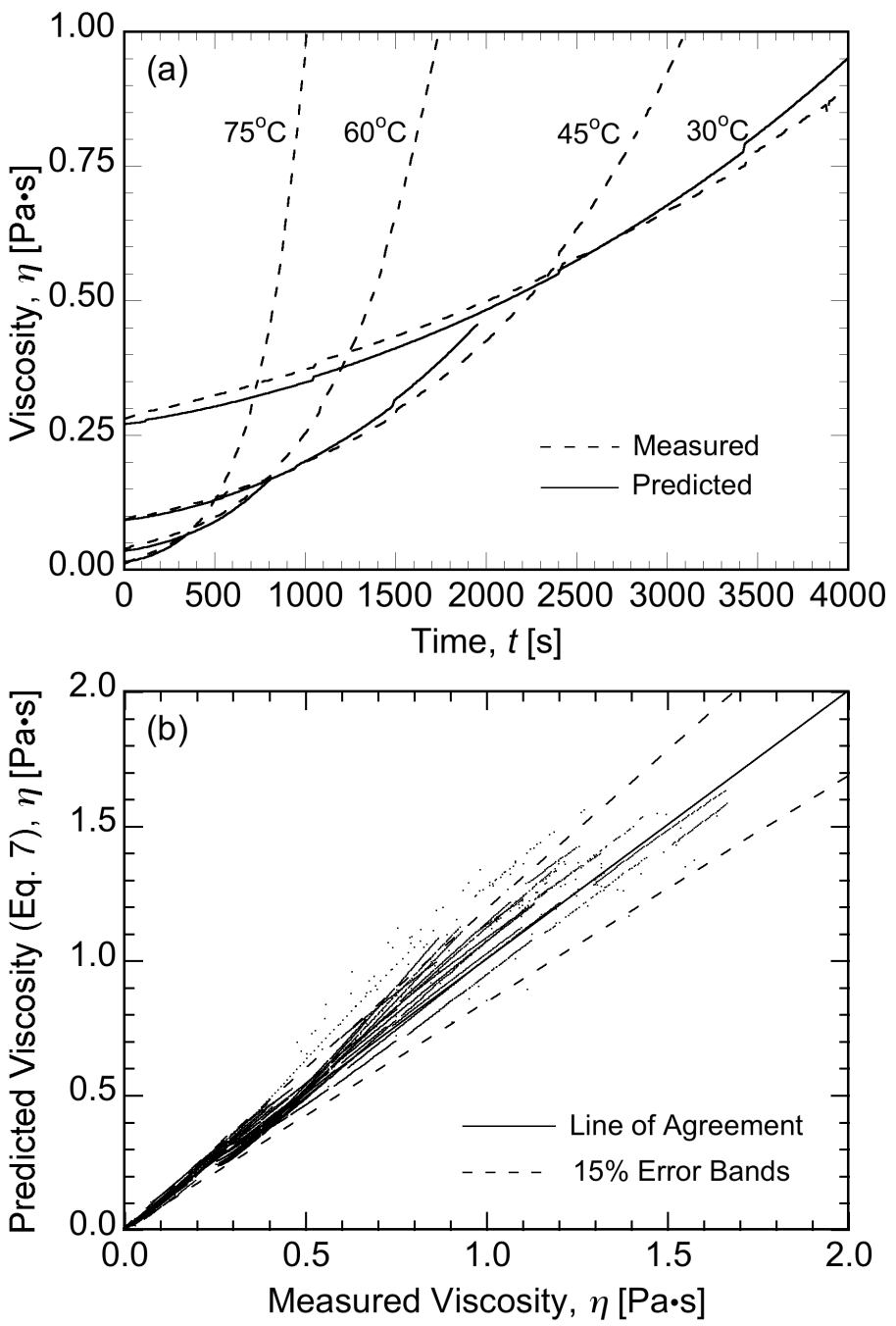

Fig. 7 (a) Validation of the generalized viscosity model, Eq. 7, with measured viscosity data for Sample 6, and (b) comparison of the model predictions with experimental data for all the cases studied.

heat of the cure reaction as well as the shape parameters of the cure kinetics expression were unaffected by the presence of the carbon nanotubes, whereas the reaction rate constant increased-specifically, through a decrease in the activation energy - with the addition of carbon nanotubes. Viscosity measurements indicated that the addition of carbon nanotubes to resin systems increases the viscosity and introduces a distinct shearthinning rheology, with the shear thinning effect being greater with increasing carbon nanotube content. Generalized models for the cure kinetics and the vicosity of the carbon nanotube filled resin system were presented and validated. The models provide a foundation for further studies on the processing of carbon-nanotube-reinforced nanocomposites.

\section{ACKNOWLEDGEMENTS}

The research was funded in part by the National Science Foundation through Grant Nos. CBET-0522933 and CBET-0934008, which is gratefully acknowledged. The authors also thank Jason Arnold, an undergraduate research assistant, for his help with the extensive experimental study.

\section{NOMENCLATURE}

$A \quad$ coefficient in the viscosity model $(\mathrm{Pa} \cdot \mathrm{s})$

$B \quad$ coefficient in the viscosity model
$E_{\eta} \quad$ activation energy in the viscosity model (J/mol)

$E \quad$ activation energy in the cure kinetics model $(\mathrm{J} / \mathrm{mol})$

$K_{0} \quad$ frequency factor in the cure kinetics model (1/s)

$K \quad$ rate constant in the cure kinetics model (1/s)

$K_{\eta} \quad$ coefficient in the generalized viscosity model

$M \quad$ consistency index in the viscosity model

$\dot{Q} \quad$ heat flow (W/g)

$R \quad$ universal gas constant $(=8.314 \mathrm{~J} / \mathrm{mol} \cdot \mathrm{K})$

$T \quad$ temperature $(\mathrm{K})$

$t \quad$ time (s)

Greek Symbols

$\Delta H_{R} \quad$ heat of the reaction $(\mathrm{J} / \mathrm{g})$

$\dot{\gamma} \quad$ shear rate $(1 / \mathrm{s})$

$\varepsilon \quad$ degree of cure

$\eta \quad$ viscosity $(\mathrm{Pa} \cdot \mathrm{s})$

$\eta_{o} \quad$ viscosity with no carbon nanotubes $(\mathrm{Pa} \cdot \mathrm{s})$

$\omega \quad$ carbon nanotube weight fraction

Subscripts/Superscripts

$m, n \quad$ shape parameters in the cure kinetics model

$n_{1} \quad$ shear-thinning exponent in the viscosity model

\section{REFERENCES}

Abdalla, M., Dean, D., Robinson, P., and Nyairo, E., 2008, "Cure behavior of epoxy/MWCNT nanocomposites: The effect of nanotube surface modification," Polymer, 49, 3310-3317, doi:10.1016/j.polymer.2008.05.016.

Advani, S., 1994, Flow and Rheology in Polymer Composites Manufacturing, Elsevier, New York.

Ajayan, P.M., Schadler, L.S., Giannaris, C., and Rubio, A., 2000, "Single-walled carbon nanotube-polymer composites: Strength and weakness," Advanced Materials, 12, 750-753, doi:10.1002/(SICI)15214095(200005)12:10<750::AID-ADMA750>3.0.CO;2-6.

Biró, L.P., Horváth, Z.E., Szalmás, L., Kertész, K., Wéber, F., Juhász, G., Radnóczi, G., and Gyulai, J., 2003, "Continuous carbon nanotube production in underwater AC electric arc," Chemical Physics Letters, 372 , 399-402, doi:10.1016/S0009-2614(03)00417-2.

Breuer, O., and Sundararaj, U., 2004, "Big returns from small fibers: A review of polymer/carbon nanotube composites," Polymer Composites, 25, 630-645, doi:10.1002/pc.20058.

Fan, Z., and Advani, S.G., 2007, "Rheology of multiwall carbon nanotube suspensions," Journal of Rheology, 51(4), 585-604, doi:10.1122/1.2736424.

Han, C.D., Lee, S.D., and Chin, H.B., 1986, "Development of a mathematical model for the pultrusion process," Polymer Engineeing Science, 26, 393-404, doi:10.1002/pen.760260604.

Hofmann, S., Kleinsorge, B., Ducati, C., Ferrari, A.C., and Robertson, J., 2004, "Low-temperature plasma enhanced chemical vapour deposition of carbon nanotubes," Diamond and Related Materials, 13, 1171-1176, doi:10.1016/j.diamond.2003.11.046.

Johnson, R.J., 2007, Processing and Performance of Composites with Micro and Nanoscale Reinforcements, Ph.D. thesis, Advanced Materials and Technologies Laboratory, University of Connecticut.

Kamal, M.R., and Sourour, S., 1973, "Kinetics and thermal characterization of thermoset cure," Polymer Engineering Science, 13, 59-64, doi:10.1002/pen.760130110. 
Kim, Y.J., Shin, T.S., Choi, H.D., Kwon, J.H., Chung, Y., and Yoon, H.G., 2005, "Electrical conductivity of chemically modified multiwalled carbon nanotube/epoxy composites," Carbon, 43, 23-30, doi:10.1016/j.carbon.2004.08.015.

Kojima, Y., Usuki, A., Kawasumi, M., Okada, A., Fukushima, Y., Kurauchi, T., and Kamigaito, O., 1993a, "Mechanical properties of nylon 6-lay hybrid," Journal of Materical Research, 8, 1185-1189, doi:10.1557/JMR.1993.1185.

Kojima, Y., USuki, A., Kawasumi, M., Okada, A., Kurauchi, T., and Kamigaito, O., 1993b, "Sorption of water in nylon 6clay hybrid," Journal of Applied Polymer Science, 49, 1259-1264, doi:10.1002/app.1993.070490715.

Koratkar, N., Suhr, J., Joshi, A., Kane, R.S., Schadler, L.S., Ajayan, P.M., and Bartolucci, S., 2005, "Characterizing energy dissipation in singlewalled carbon nanotube polycarbonate composites," Applied Physical Letters, 87, 063102, doi:10.1063/1.2007867.

Koratkar, N., Wei, B.Q., and Ajayan, P.M., 2002, "Carbon nanotube films for damping applications," Advanced Materials, 14, 997-1000, doi:10.1002/1521-4095(20020705)14:13/14<997::AIDADMA997>3.0.CO;2-Y.

Kordon, A.K., Pitchumani, R., Beris, A.N., Karbhari, V.M., and Dhurjati, P.S., 1993, "A rheological model for particulate caramic slurries at low temperatures," Scripta Metallurgica et Materiala, 29, 1095-1099, doi:10.1016/0956-716X(93)90184-T.

Lee, W.I., Loos, A.C., and Springer, G.S., 1982, "Heat of Reaction, Degree of Cure, and Viscosity of Hercules 3501-6 Resin," Composite Materials, 16, 510-520, doi:10.1177/002199838201600605.

Li, X., Gao, H., Scrivens, W.A., Fei, D., Thakur, V., Sutton, M.A., Reynolds, A.P., and Myrick, M.L., 2005, "Structural and mechanical characterization of nanoclay-reinforced agarose nanocomposites," Nanotechnology, 16, 2020-2029, doi:10.1088/0957-4484/16/10/006.

Nielsen, L.E., and Landel, R.F., 1994, Mechanical Properties of Polymers and Composites, Marcel Dekker, New York.

Njuguna, J., and Peilichowski, K., 2004, "Polymer nanocomposites for aerospace applications: Fabrication," Advanced Engineering Materials, 6, 193-203, doi:10.1002/adem.200305111.

Ohshima, S., Ago, H., Inoue, H., and Yumura, M., 2001, “Development of mass-production technology for multi-walled carbon nanotubes," New Diamond and Frontier Carbon Technology, 11, 437-448.

Opaliki, M., Kenny, J.M., and Nicolais, L., 1996, "Cure kinetics of neat and carbon-fiber-reinforced TGDDM/DDS epoxy systems," Journal of Applied Polymer Science, 61, 1025-1037, doi:10.1002/(SICI)10974628(19960808)61:6<1025::AID-APP17>3.0.CO;2-V.

Rajoria, H., and Jalili, N., 2005, "Passive vibration damping enhancement using carbon nanotube-epoxy reinforced composites," Composite Science and Technology, 65, 2079-2093, doi:10.1016/j.compscitech.2005.05.015.

Ramakrishnan, B., Zhu, L., and Pitchumani, R., 2000, "Curing of composites using internal resistive heating," ASME Journal of Manufacturing Science and Engineering, 122, 124-131, doi:10.1115/1.538913.
Schadler, L.S., Giannaris, S.C., and Ajayan, P.M., 1998, "Load transfer in carbon nanotube epoxy composites," Applied Physics Letters, 73, 38423844, doi:10.1063/1.122911.

Smith Jr., J.G., Delozier, D.M., Connell, J.W., and Watson, K.A., 2004, "Carbon nanotube-conductive additive-space durable polymer nanocomposite films for electrostatic charge dissipation," Polymer, 45, 6133-6142, doi:10.1016/j.polymer.2004.07.004

Terenzi, A., Vedova, C., Lelli, G., Mijovic, J., Torre, L., Valentini, L., and Kenny, J.M., 2008, "Chemorheological behavior of double-walled carbon nanotube-epoxy nanocomposites," Composites Science and Technology, 68, 1862-1868, doi:10.1016/j.compscitech.2008.01.005.

Ton-That, M.T., Ngo, T.D., Ding, P., Fang, G., Cole, K.C., and Hoa, S.V., 2004, "Epoxy nanocomposites: Analysis and kinetics of cure," Polymer Engineering Science, 44, 1132-1141, doi:10.1002/pen.20106.

Velasco-Santos, C., Martínez-Hernández, A.L., Fisher, F.T., Ruoffa, R., and no, V.M.C., 2003, "Improvement of thermal and mechanical properties of carbon nanotube composites through chemical functionalization," Chemical Materials, 15, 4470-4475, doi:10.1021/cm034243c.

Wang, S., Liang, Z., Liu, T., Wang, B., and Zhang, C., 2006, "Effective amino-functionalization of carbon nanotubes for reinforcing epoxy polymer composites," Nanotechnology, 17, 1551-1557, doi:10.1088/0957$4484 / 17 / 6 / 003$

Wang, Y., Wei, F., Luo, G., Yu, H., and Gu, G., 2002, “The large-scale production of carbon nanotubes in a nano-agglomerate fluidized-bed reactor," Chemical Physics Letters, 364, 568-572, doi:10.1016/S00092614(02)01384-2.

Xie, H., Liu, B., Sun, Q., Yuan, Z., Shen, J., and Cheng, R., 2005, "Cure kinetic study of carbon nanofibers/epoxy composites by isothermal DSC," Journal of Applied Polymer Science, 96, 329-335, doi:10.1002/app.21415.

Xie, H., Liu, B., Yang, H., Wang, Z., Shen, J., and Cheng, R., 2006, "Thermal characterization of carbon-nanofiber-reinforced tetraglycidyl4,4'-diaminodiphenylmethane/4,4'-diaminodiphenylsulfone epoxy composites," Journal of Applied Polymer Science, 100, 295-298, doi:10.1002/app.23106.

Xie, H., Liu, B., Yuan, Z., Shen, J., and Cheng, R., 2004, "Cure kinetics of carbon nanotube/tetrafunctional epoxy nanocomposites by isothermal differential scanning calorimetry," Journal of Polymer Science Part B: Polymer Physics, 42, 3701-3712, doi:10.1002/polb.20220.

Xu, Y., Ray, G., and Abdel-Magid, B., 2006, "Thermal behavior of single-walled carbon nanotube polymer matrix composites," Compos Part A, 37, 114-121, doi:10.1016/j.compositesa.2005.04.009.

Zhamu, A., Jana, S., Salehi-Khojin, A., Kolodka, E., Gan, Y.X., and Zhong, W.H., 2007, "Chemorheology of reactive graphite nanofiberreinforced epoxy as composite matrix," Composite Intefaces, 14, 177_ 198, doi:10.1163/156855407780340331.

Zhou, X., Shin, E., Wang, K.W., and Bakis, C.E., 2004, "Interfacial damping characteristics of carbon nanotube-based composites," Composite Science and Technology, 64, 2425-2437, doi:10.1016/j.compscitech.2004.06.001. 